\title{
Factors associated with hepatic dysfunction in hepatitis B-positive patients with postgastrectomy adenocarcinoma
}

\author{
JIAN XU*, HONG ZHU* , YAQIN ZHAO, XIN WANG, YALI SHEN, WU WANG and FENG XU \\ Department of Radiation Oncology, Cancer Center, West China Hospital, Sichuan University, Chengdu, Sichuan, P.R. China
}

Received January 24, 2012; Accepted May 23, 2012

DOI: $10.3892 / \mathrm{ol} .2012 .745$

\begin{abstract}
In the present study, we reviewed 44 postgastrectomy adenocarcinoma patients who had hepatitis $\mathrm{B}$ and received treatment in the Abdominal Cancer Department of the West China Hospital between October 2006 and October 2010. Of these patients, 17 developed hepatic dysfunction. Radiotherapy is an independent risk factor to hepatic function on univariate and multivariate analysis. Grade III or IV hepatic dysfunction was developed by five patients, all of whom had received radiotherapy and had reactivated hepatic $\mathrm{B}$ virus (HBV). Radiotherapy is a significant risk factor to hepatic function in patients with postgastrectomy adenocarcinoma carrying HBV, thus we suggest excluding the liver from the radiation field. $\mathrm{HBV}$ reactivation plays a role in the development of grade III or IV hepatic dysfunction. Patients with reactivated HBV should immediately receive regular antiviral treatment.
\end{abstract}

\section{Introduction}

Hepatic function is one of the most significant indices for cancer patients who undergo chemoradiation treatment $(1,2)$. The patients were required to take a biochemical test prior to radiotherapy or chemotherapy. Patients with hepatic dysfunction were not permitted to continue the treatment.

Hepatitis B is a common infectious disease in Asia, particularly in China (3-5). Approximately 10\% of the Chinese population are hepatitis B virus (HBV) carriers and numerous patients are likely to develop hepatic cirrhosis and liver cancer $(6,7)$. A significant proportion of hepatic cancer patients also present with hepatitis $\mathrm{B}$; however, there are numerous patients with non-hepatic cancer who also carry hepatitis B in China. The latter patients were also found to be

Correspondence to: Professor Feng Xu, Department of Radiation Oncology, Cancer Center, West China Hospital, Sichuan University, Guoxue Road 37, Chengdu 610041, P.R. China

E-mail: fengxuster@gmail.com

${ }^{*}$ Contibuted equally

Key words: hepatic dysfunction, radiotherapy, hepatitis B virus, gastric cancer, hepatitis B virus reactivation more likely to develop hepatic dysfunction during the process of radiotherapy or chemotherapy (8-11).

Previously, numerous studies focused on patients with lymphoma and hepatitis B treated with rituximab (12-14). Few studies investigated other patients with other types of cancer with hepatitis B. Cheng et al (1) studied 62 postgastrectomy patients treated with concomitant chemoradiotherapy (CCRT) and observed that HBV infection was the only independent factor associated with chemoradiation-induced liver disease. However, only 11 patients were HBV carriers and all the patients were treated with postgastrectomy CCRT. Since hepatic function is extremely important for cancer patients, this study aims to investigate the factors associated with hepatic function in a larger population of postgastrectomy cancer patients carrying HBV.

\section{Patients and methods}

Patient population and characteristics. Patients with stage IB-IV $\mathrm{M}_{0}$ gastric adenocarcinoma carrying HBV who underwent total or subtotal gastrectomy and regional lymph node dissection and were treated in the Abdominal Cancer Department of the West China Hospital (Chengdu, China) between October 2006 and October 2010 were reviewed. The clinical staging system of gastric cancer (AJCC 2005 version) was adopted for this study. This study was approved by the Ethics Committee of Sichuan University. All patients gave informed consent to participate in the study. Patients who had hepatic dysfunction, HBV activation or hepatic cirrhosis prior to treatment were excluded from this study. Patients who failed to complete the radiotherapy for reasons other than hepatic dysfunction or HBV activation were also excluded. In total 44 patients remained who were confirmed to have gastric adenocarcinoma by the Pathological Department of our hospital. The patients were diagnosed as HBV carriers by the Laboratory Department of our hospital with serum HBV DNA $<5 \mathrm{pg} / \mathrm{ml}$. Patients with grade I hepatic dysfunction who recovered soon after completing treatment were also included. The characteristics of these patients are listed in Table I.

Chemotherapy. A total of 44 gastric cancer patients received postgastrectomy chemotherapy: CF, FOLFIRI, XELOX and EOF regimens were each used in four patients; the $\mathrm{PTX}+\mathrm{DDP}+5-\mathrm{F}$ protocol was used in two; the ECF regimen 
Table I. Patient characteristics $(n=44)$.

\begin{tabular}{lr}
\hline Patient characteristics & No. of patient \\
\hline Discrete variables & \\
Gender & \\
Male & $28(63.64)$ \\
Female & $16(36.36)$ \\
AJCC stage & \\
IB & $4(9.09)$ \\
II & $7(15.91)$ \\
III & $18(40.91)$ \\
IV & $15(34.09)$ \\
Radiotherapy & \\
Yes & $17(38.64)$ \\
No & $27(61.36)$ \\
Chemotherapy cycles & \\
$<6$ & $26(59.09)$ \\
$\geq 6$ & $18(40.91)$
\end{tabular}

Continuous variables

Age (years), median (range)

$56(35-73)$

AJCC, American Joint Committee on Cancer.

was used in three patients; mFOLFOX 6, mFOLFOX 7 or FOLFOX 4 regimens were used in the remaining patients. For the patients who received CCRT, the dosage of chemotherapy was reduced by $0-20 \%$ following the start of radiotherapy according to the reaction of the patient.

Radiotherapy. A total of 16 patients received CCRT. In general, the radiotherapy began with the second cycle of chemotherapy. Intensity modulated radiotherapy (IMRT) or image-guided radiotherapy (IGRT) were used in all the patients. The radiation techniques were introduced in previous studies (15). Briefly, the patients were immobilized in the Stereotactic Body Frame (Elekta, Stockholm, Sweden), which uses a vacuum pillow in a rigid frame, with an abdominal compression device. CT-guided simulation was performed and the gross tumor volume (GTV) was defined. The target and normal adjacent structures were contoured on the planning CT scan. Multileaf collimator (MLC) blocking was used to block normal tissues outside of the intended targeted tissues. Treatment was delivered once daily with 1.8-2.0 Gy, five fractions per week by a 6-MV linear accelerator. For the patients who received CCRT, the median dose was 50.4 Gy (range, 45-50.4). The clinical target volume (CTV) included the preoperative stomach volume, surgical bed, gastric remnant and perigastric lymph nodes. Other lymph node areas, including mediastinal, porta hepatis, splenic hilum, pancreaticoduodenal and peripancreatic, were included if they were at risk based on the primary tumor location or pathological involvement of the lymph nodes. With regard to the bowel, the intestinal loops outside the planning treatment volume (PTV) were contoured, but not the whole abdominal space. To account for daily setup error and organ motion, the

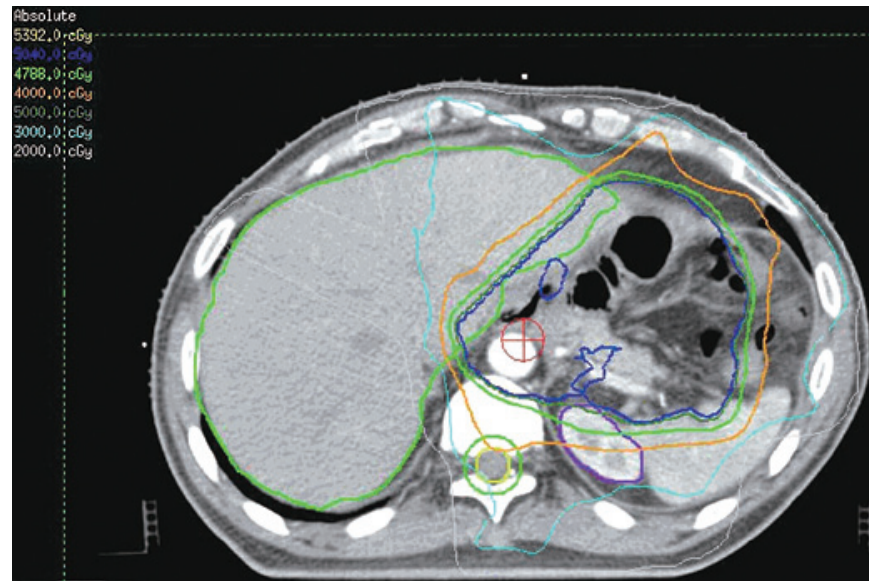

Figure 1. CTV delineation of one representative patient treated with the IMRT technique. CTV, clinical target volume; IMRT, intensity modulated radiotherapy.

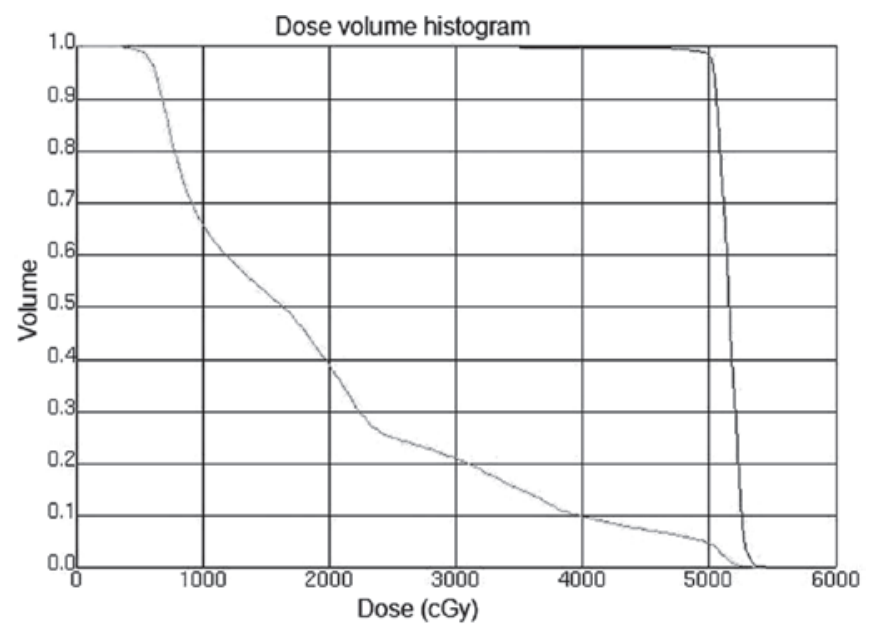

Figure 2. Dose volume histograms (DVHs) for the PTV of one representative patient treated with the IMRT technique. PVT, planning treatment volume; IMRT, intensity modulated radiotherapy.

CTV to PTV expansion was typically 5-10 mm. Normal structures were also contoured, including kidneys, liver, spinal cord and bowel. The mean hepatic dose and dose to $30 \%$ volume of liver (V30) was maintained at $<30$ Gy (V30<30\%). Figs. 1 and 2 show the CTV delineation and dose volume histograms (DVHs) for the PTV of one representative patient treated with the IMRT technique, respectively.

Follow-up. The patients were examined weekly during the treatment period, involving taking patient history, physical examination, blood test and biochemical test, and were followed up every month after finishing the treatment for at least 3 months. HBV DNA copies were examined at the beginning of the treatment and repeated for the patients who had been examined for hepatic dysfunction during the treatment. The cases of hepatic dysfunction were recorded according to the WHO guideline and levels of serum HBV DNA $>5 \mathrm{pg} / \mathrm{ml}$ were defined as HBV reactivation (1). The patients who had serious hepatic dysfunction had received corresponding treatment, including tiopronin, glutathione, multi-phosphatidylcholine 
Table II. Characteristics of patients with hepatic dysfunction $(n=17)$.

\begin{tabular}{lc}
\hline Patient characteristics & No. of patients $(\%)$ \\
\hline Discrete variables & \\
Gender & \\
Male & $13(76.47)$ \\
Female & $4(23.53)$ \\
AJCC stage & \\
IB & $0(0)$ \\
II & $4(23.53)$ \\
III & $10(58.82)$ \\
IV & $3(17.65)$ \\
Radiotherapy & \\
Yes & $12(70.59)$ \\
No & $5(29.41)$ \\
Chemotherapy cycles & \\
$<6$ & $8(47.06)$ \\
$\geq 6$ & $9(52.94)$ \\
Virus reactivation & \\
Yes & \\
No & \\
Continuous variables & $9(47.06)$ \\
Age (years), median (range) & \\
\hline
\end{tabular}

AJCC, American Joint Committee on Cancer.

and vitamin B. Patients who had HBV DNA reactivation had received lamivudine treatment for at least half a year, besides which, the patients did not receive any other treatments. All 44 patients completed treatment by July 2011.

Statistical analysis. The SPSS software program (version 16.0; SPSS Inc., Chicago, IL, USA) was used for all statistical analyses. For the univariate analysis, a Student's t-test was used to compare continuous variables between the patients with or without hepatic dysfunction and a Chi-square test was used to compare discrete variables. Logistic regression analysis was used to investigate the multivariable impact on the occurrence of hepatic dysfunction. $\mathrm{P}<0.05$ was considered to indicate a statistically significant result.

\section{Results}

Patient characteristics. Following treatment, 17 patients developed hepatic dysfunction. The characteristics of these patients are listed in Table II. HBV had reactivated in eight out of 17 patients, five of whom had received CCRT. Findings of the univariate analysis showed that only radiotherapy was a significant associating factor of patients who had developed hepatic dysfunction $(\mathrm{P}=0.001)$. Other factors that were not statistically significant include gender $(\mathrm{P}=0.165)$, age $(\mathrm{P}=0.393)$, AJCC stage $(\mathrm{P}=0.377)$ and chemotherapy cycles $(\mathrm{P}=0.203$; Table III $)$. Results of the multivariate analysis
Table III. Univariate analysis of patients who developed hepatic dysfunction.

\begin{tabular}{lcc}
\hline Patient characteristics & No. of patients (\%) & P-value \\
\hline Discrete variables & & \\
Gender & & \\
Male & $28(63.64)$ & 0.165 \\
Female & $16(36.36)$ & \\
AJCC stage & & \\
IB & $4(9.09)$ & 0.377 \\
II & $7(15.91)$ & \\
III & $18(40.91)$ & \\
IV & $15(34.09)$ & \\
Radiotherapy & & \\
Yes & $17(38.64)$ & \\
No & $27(61.36)$ & \\
Chemotherapy cycles & & \\
$<6$ & $26(59.09)$ & 0.203 \\
$\geq 6$ & $18(40.91)$ & \\
Continuous variables & & \\
Age (years), median (range) & $56(35-73)$ & \\
\hline AJCC, American Joint Committee on Cancer. & \\
\hline
\end{tabular}

showed that only radiotherapy was a significant associating factor for patients who had also developed hepatic dysfunction [odds ratio, 10.560; 95\% confidence interval (CI), 2.539-43.918; $\mathrm{P}=0.001$; Table IV].

Dosimetric characteristics. To determine the internal dosimetric correlation with hepatic dysfunction, we listed the dosimetric characteristics of the 16 patients who received radiotherapy, including normal liver volume, isocenter dose, V30 and mean liver dose (mean $\pm \mathrm{SD}$; Table V). A Student's t-test was used to analyze the correlation between the dosimetric characteristics and hepatic dysfunction and we observed that mean liver dose was a significant associating factor $(\mathrm{P}=0.002$; Table $\mathrm{V})$.

Severe hepatic dysfunction. A total of five patients developed severe (grade III or IV) hepatic dysfunction in the present study (Table VI). The five patients were male, aged 35-71 years old and the AJCC stages were either stage II or IIIb. The patients had received radiotherapy and the number of chemotherapy cycles ranged from 4 to 7 , including EOF, mFOLFOX6 and mFOLFOX 7 regimens. In these five patients, the hepatic dysfunctions were mainly enhanced aminotransferase levels, including alanine aminotransferase (ALT) and aspartate aminotransferase (AST). The bilirubins appeared almost normal. The HBV was reactivated in these five patients, all of whom recovered following lamivudine treatment.

\section{Discussion}

Chronic HBV infection is a major public health problem in Asian countries and the reactivation of $\mathrm{HBV}$ prior to or 
Table IV. Multivariate analysis of patients who developed hepatic dysfunction.

\begin{tabular}{lcccccccr}
\hline Factor & & & & & & & \multicolumn{2}{c}{$95 \%$ CI for $\operatorname{Exp}(\mathrm{B})$} \\
\hline Radiotherapy & 2.357 & 0.727 & 10.507 & 1 & 0.001 & 10.560 & 2.539 & 43.918 \\
\hline
\end{tabular}

CI, confidence interval. B, regression coefficient; SE, standard of error; Wald, X2 value; Sig., P-value; Exp(B), relative risk.

Table V. Dosimetric characteristics and univariate analysis (Student's t-test) of patients who received radiotherapy $(n=16)$.

\begin{tabular}{lcc}
\hline Variable & Mean \pm SD & P-value \\
\hline Normal liver volume (ml) & $1266 \pm 284$ & 0.834 \\
Isocenter dose (Gy) & $50.7 \pm 2.8$ & 0.198 \\
V30 (\%) & $26.4 \pm 2.4$ & 0.730 \\
Mean liver dose (cGy) & $2133 \pm 139$ & 0.002 \\
\hline
\end{tabular}

SD, standard deviation; Gy, grays; V30, volume fraction of normal liver that received a radiation dose $<30 \mathrm{~Gy}$.

following treatment with immunosuppressive or anticancer agents has been documented since $1975(6,16)$. The most commonly reported types of chemotherapy associated with HBV reactivation are those used for the treatment of hematological malignancies, including acute leukemia, myeloproliferative disorders, lymphoproliferative disorders and plasma cell dyscrasias (17-19). One of the most frequently studied examples is HBV reactivation in lymphoma treated with rituximab since it is able to specifically induce the apoptosis of B lymphocytes and leads to a marked immunosuppression in patients. However, few studies concerning the effect of chemotherapy on HBV reactivation in other cancer patients, including gastric cancer, have been performed.

Radiotherapy, as a locoregional treatment, may also cause human immunosuppression $(1,11,20,21)$. Therefore radiotherapy may affect $\mathrm{HBV}$ reactivation in the treatment of cancer patients, especially in patients with liver located in the radiation field. Cheng et al (11) compared $65 \mathrm{HBV}$-related hepatocellular cancer (HCC) patients and 24 other HCC patients treated with three-dimensional conformal radiotherapy (3D-CRT) and found that the HCC patients who were HBV carriers presented with a statistically significantly greater susceptibility to radiation-induced liver disease following 3D-CRT. Kim et al (22)

Table VI. Characteristics of five patients who developed grade III or IV hepatic dysfunction.

Patient no.

\begin{tabular}{|c|c|c|c|c|c|}
\hline \multirow[b]{2}{*}{ Characteristics } & \\
\hline & 1 & 2 & 3 & 4 & 5 \\
\hline Gender & M & M & M & M & M \\
\hline Age (years) & 43 & 41 & 43 & 35 & 71 \\
\hline AJCC stage & $\mathrm{IIIb}$ & II & $\mathrm{IIIb}$ & II & IIIb \\
\hline Radiotherapy & Yes & Yes & Yes & Yes & Yes \\
\hline Chemo. regimens & mFOLFOX6 & $\mathrm{EOF}$ & mFOLFOX7 & mFOLFOX7 & mFOLFOX7 \\
\hline Chemo. cycles & 7 & 4 & 5 & 7 & 5 \\
\hline Isocenter dose (Gy) & 50.4 & 50.4 & 50.4 & 50.4 & 50.4 \\
\hline Liver volume (ml) & 1297 & 875 & 1410 & 1177 & 1420 \\
\hline Mean liver dose (cGy) & 2137 & 2313 & 2181 & 2164 & 2191 \\
\hline $\mathrm{V} 30(\%)$ & 26 & 22 & 24 & 25 & 27 \\
\hline ALT (IU/1) & 538 & 245 & 326 & 341 & 240 \\
\hline AST (IU/l) & 315 & 265 & 294 & 209 & 210 \\
\hline Tbil $(\mu \mathrm{mol} / \mathrm{l})$ & $\mathrm{N}$ & $\mathrm{N}$ & $\mathrm{N}$ & $\mathrm{N}$ & $\mathrm{N}$ \\
\hline Dbil $(\mu \mathrm{mol} / \mathrm{l})$ & 9.1 & $\mathrm{~N}$ & 9.6 & $\mathrm{~N}$ & $\mathrm{~N}$ \\
\hline HBV DNA reactivation & Yes & Yes & Yes & Yes & Yes \\
\hline Lamivudine treatment & Yes & Yes & Yes & Yes & Yes \\
\hline Outcome of dysfunction & $\mathrm{R}$ & $\mathrm{R}$ & $\mathrm{R}$ & $\mathrm{R}$ & $\mathrm{R}$ \\
\hline
\end{tabular}

M, male; AJCC, American Joint Committee on Cancer; Chemo., chemotherapy; Gy, grays; V30, the volume fraction of the normal liver that received a radiation dose $<30 \mathrm{~Gy}$; ALT, alanine aminotransferase; AST, aspartate aminotransferase; Tbil, total bilirubin; N, normal; Dbil, direct bilirubin; $\mathrm{R}$, recovery. 
compared $32 \mathrm{HBV}$-related HCC patients treated with 3D-CRT and $43 \mathrm{HBV}$-related $\mathrm{HCC}$ patients without any treatment and observed that the cumulative rate of HBV reactivation was significantly greater in the treatment group. In the radiotherapy treatment of gastric cancer, part of the liver is located in the radiation field. Investigation into the influence of radiotherapy on HBV reactivation in gastric cancer patients is needed.

Chemoradiation treatment is unable to cause HBV reactivation in all the cancer patients who have hepatitis $\mathrm{B}(16,23)$ and the patients who did not develop HBV reactivation may also have hepatic dysfunction. Therefore, we studied the associating factors of hepatic function in 44 postgastrectomy patients who carried HBV and were treated in our department.

In the present study, 17 patients developed hepatic dysfunction, five of whom were of grade III or IV. The five patients had received CCRT, thus of the total 16 patients that received CCRT, the rate of patients that developed grade III or IV hepatic dysfunction was $31.25 \%(5 / 16)$. This rate is lower than that observed in the study by Cheng et al (1), which was $54.55 \%(6 / 11)$. In the present study, HBV was reactivated in eight patients, five of whom had received CCRT, thus of the 16 patients that received CCRT, the rate of patients who had HBV reactivated was $31.25 \%(5 / 16)$ in comparison to the incidence rate of $36.36 \%$ (4/11) in the study by Cheng et al (1). Therefore the study by those authors (1) had a higher severe hepatic dysfunction rate and HBV reactivation rate than our study. When comparing the treatment differences between the present study and that by Cheng et al, we observed that although we had used the IMRT technique, the rates of the isocenter dose, dose to the liver and V30 were higher in our study than those reported Cheng et al (1). Therefore, we suggest that this difference is due to the majority of the patients from the study by Cheng et al undergoing neoadjuvant chemotherapy (93.55\%), which none of our patients received. However, of the $51 \mathrm{HBV}$-negative postgastrectomy patients who received CCRT in the study by Cheng et al (1), the rate of grade III or IV hepatic dysfunction was $3.92 \%(2 / 51)$, which was markedly lower than the incidence rate in the present study. Therefore, we suggest that $\mathrm{HBV}$ is a significant factor that causes severe hepatic dysfunction.

In the univariate and multivariate analyses, radiotherapy was the only significant associating factor of hepatic function in the 44 patients. In the study by Cheng et al (1), radiotherapy was not suggested to be an associating factor since their patients had received CCRT. In the present study, chemotherapy cycle was not found to be a significant associating factor which was similar to the findings of Cheng et al (1). However, whether chemotherapy or different chemotherapy regimens were significant associating factors requires further investigation since patients in the current study had received postgastrectomy chemotherapy and most of the chemotherapy regimens were similar. Although radiotherapy was an independent risk factor of hepatic function, it remains unclear whether radiation to the liver was the main cause. However, we suggest excluding the liver from radiation field.

Since radiotherapy was the only significant associating factor of hepatic function in this study, we studied the dosimetric difference of 16 patients who had received CCRT. The characteristics of these 16 patients are listed in Table $\mathrm{V}$ and we observed that the mean liver dose was a significant associating factor of hepatic function using the Student's t-test $(\mathrm{P}=0.002)$. However, there were only 16 patients who had received radiotherapy, thus it is difficult to reach a firm conclusion.

The five patients who developed grade III or IV hepatic dysfunction received CCRT and also had HBV reactivated. In the study by Cheng et al (1), the HBV status was the only significant associating factor correlated with the grade III or IV hepatic dysfunction. Therefore we suggest that HBV reactivation played a significant role in the development of serious hepatic dysfunction. The major abnormalities of these five patients were aminotransferase activity although the bilirubin levels were rarely affected.

In the present study, the patients who had HBV reactivated had received lamivudine treatment regularly and recovered following treatment. $\mathrm{HBV}$ reactivation is a serious complication for hepatitis $\mathrm{B}$ patients and can occasionally be fatal $(10,24,25)$, therefore it is vital to take regular antiviral treatment. Lamivudine is the most commonly used drug for HBV reactivation $(26,27)$. However, results of numerous studies have indicated that entecavir may be more effective (28-30).

In conclusion, radiotherapy was an independent risk factor of hepatic function in postgastrectomy adenocarcinoma patients positive for HBV. We suggest excluding the liver from the radiation field. $\mathrm{HBV}$ is important in the development of severe hepatic dysfunction and patients in whom HBV has reactivated should take immediate and regular antiviral treatment.

\section{Acknowledgements}

This study was supported by grants from the National Basic Research Program of China (No. 2011CB935800).

\section{References}

1. Cheng JC, Liu MC, Tsai SY, Fang WT, Jer-Min Jian J and Sung JL: Unexpectedly frequent hepatitis B reactivation by chemoradiation in postgastrectomy patients. Cancer 101: 2126-2133, 2004

2. Stange MA, Tutarel O, Pischke S, Schneider A, Strassburg CP, Becker T, Barg-Hock H, Basturk M, Wursthorn K, Cornberg M, et al: Fulminant hepatic failure due to chemotherapy-induced hepatitis B reactivation: role of rituximab. Z Gastroenterol 48: 258-263, 2010.

3. Lubel JS and Angus PW: Hepatitis B reactivation in patients receiving cytotoxic chemotherapy: diagnosis and management. J Gastroenterol Hepatol 25: 864-871, 2010.

4. Koo YX, Tan DS, Tan IB, Tao M, Chow WC and Lim ST: Hepatitis B virus reactivation and role of antiviral prophylaxis in lymphoma patients with past hepatitis B virus infection who are receiving chemoimmunotherapy. Cancer 116: 115-121, 2010.

5. Lim SM, Jang JW, Kim BW, Choi H, Choi KY, Park SJ and Han CW: Hepatitis B virus reactivation during chlorambucil and prednisolone treatment in an HBsAg-negative and antiHBs-positive patient with B-cell chronic lymphocytic leukemia. Korean J Hepatol 14: 213-218, 2008 (In Korean).

6. Wu JM, Huang YH, Lee PC, Lin HC and Lee SD: Fatal reactivation of hepatitis $B$ virus in a patient who was hepatitis B surface antigen negative and core antibody positive before receiving chemotherapy for non-Hodgkin lymphoma. J Clin Gastroenterol 43: 496-498, 2009

7. Liu CJ, Chen PJ, Chen DS and Kao JH: Hepatitis B virus reactivation in patients receiving cancer chemotherapy: natural history, pathogenesis, and management. Hepatol Int 14: 14, 2011.

8. Yagci M, Ozkurt ZN, Yegin ZA, Aki Z, Sucak GT and Haznedar R: Hepatitus B virus reactivation in HBV-DNA negative and positive patients with hematological malignancies. Hematology 15: 240-244, 2010. 
9. Zhang B, Wang J, Xu W, Wang L and Ni W: Fatal reactivation of occult hepatitis $B$ virus infection after rituximab and chemotherapy in lymphoma: necessity of antiviral prophylaxis. Onkologie 33: 537-539, 2010.

10. Day FL, Karnon J and Rischin D: Cost-effectiveness of universal hepatitis B virus screening in patients beginning chemotherapy for solid tumors. J Clin Oncol 29: 3270-3277, 2011.

11. Cheng JC, Wu JK, Lee PC, Liu HS, Jian JJ, Lin YM, Sung JL and Jan GJ: Biologic susceptibility of hepatocellular carcinoma patients treated with radiotherapy to radiation-induced liver disease. Int J Radiat Oncol Biol Phys 60: 1502-1509, 2004

12. Evens AM, Jovanovic BD, Su YC, Raisch DW, Ganger D, Belknap SM, Dai MS, Chiu BC, Fintel B, Cheng Y, et al: Rituximab-associated hepatitis $B$ virus (HBV) reactivation in lymphoproliferative diseases: meta-analysis and examination of FDA safety reports. Ann Oncol 22: 1170-1180, 2011.

13. Matsue K, Kimura S, Takanashi Y, Iwama K, Fujiwara H, Yamakura M and Takeuchi M: Reactivation of hepatitis B virus after rituximab-containing treatment in patients with CD20positive B-cell lymphoma. Cancer 116: 4769-4776, 2010.

14. Niitsu N, Hagiwara Y, Tanae K, Kohri M and Takahashi N: Prospective analysis of hepatitis B virus reactivation in patients with diffuse large B-cell lymphoma after rituximab combination chemotherapy. J Clin Oncol 28: 5097-5100, 2010.

15. Minn AY, Hsu A, La T, Kunz P, Fisher GA, Ford JM, Norton JA, Visser B, Goodman KA, Koong AC and Chang DT: Comparison of intensity-modulated radiotherapy and 3-dimensional conformal radiotherapy as adjuvant therapy for gastric cancer. Cancer 116: 3943-3952, 2010

16. Ji D, Cao J, Hong X, Li J, Wang J, Chen F, Wang C and Zou S: Low incidence of hepatitis $\mathrm{B}$ virus reactivation during chemotherapy among diffuse large B-cell lymphoma patients who are HBsAg-negative/HBcAb-positive: a multicenter retrospective study. Eur J Haematol 85: 243-250, 2010.

17. Roche B and Samuel D: The difficulties of managing severe hepatitis B virus reactivation. Liver Int 31 (Suppl 1): 104-110, 2011.

18. Artz AS, Somerfield MR, Feld JJ, Giusti AF, Kramer BS, Sabichi AL, Zon RT and Wong SL: American Society of Clinical Oncology provisional clinical opinion: chronic hepatitis B virus infection screening in patients receiving cytotoxic chemotherapy for treatment of malignant diseases. J Clin Oncol 28: 3199-3202, 2010.

19. Marinone $C$ and Mestriner M: HBV disease: HBsAg carrier and occult B infection reactivation in haematological setting. Dig Liver Dis 43 (Suppl 1): S49-56, 2011.
20. Chou CH, Chen PJ, Jeng YM, Cheng AL, Huang LR and Cheng JC: Synergistic effect of radiation and interleukin-6 on hepatitis B virus reactivation in liver through STAT3 signaling pathway. Int J Radiat Oncol Biol Phys 75: 1545-1552, 2009.

21. Cheng JC, Liu HS, Wu JK, Chung HW and Jan GJ: Inclusion of biological factors in parallel-architecture normal-tissue complication probability model for radiation-induced liver disease. Int $\mathrm{J}$ Radiat Oncol Biol Phys 62: 1150-1156, 2005.

22. Kim JH, Park JW, Kim TH, Koh DW, Lee WJ and Kim CM Hepatitis B virus reactivation after three-dimensional conformal radiotherapy in patients with hepatitis $B$ virus-related hepatocellular carcinoma. Int J Radiat Oncol Biol Phys 69: 813-819, 2007.

23. Ide Y, Ito Y, Takahashi S, Tokudome N, Kobayashi K, Sugihara T, Hattori M, Yokoyama M, Uchiyama A, Inoue K, et al: Hepatitis B virus reactivation in adjuvant chemotherapy for breast cancer. Breast Cancer Jul 24, 2010 (Epub ahead of print).

24. Kusumoto S, Tanaka Y, Mizokami M and Ueda R: Clinical significance of hepatitis B virus (HBV)-DNA monitoring to detect HBV reactivation after systemic chemotherapy. J Clin Oncol 29: e100; author reply e101, 2011.

25. Day FL, Link E, Thursky K and Rischin D: Current hepatitis B screening practices and clinical experience of reactivation in patients undergoing chemotherapy for solid tumors: a nationwide survey of medical oncologists. J Oncol Pract 7: 141-147, 2011.

26. Huang H, Cai Q, Lin T, Lin X, Liu Y, Gao Y and Peng R: Lamivudine for the prevention of hepatitis $\mathrm{B}$ virus reactivation after high-dose chemotherapy and autologous hematopoietic stem cell transplantation for patients with advanced or relapsed non-Hodgkin's lymphoma single institution experience. Expert Opin Pharmacother 10: 2399-2406, 2009.

27. You CR, Jang JW, Choi JK, Bae SH, Yoon SK, Kay CS and Choi JY: Hepatic failure caused by reactivation of YMDD mutants occurring during preemptive lamivudine therapy. Gut Liver 4: 262-265, 2010.

28. Colson P, Borentain P, Coso D, Chabannon C, Tamalet C and Gérolami R: Entecavir as a first-line treatment for HBV reactivation following polychemotherapy for lymphoma. Br J Haematol 143: 148-150, 2008.

29. Brost S, Schnitzler P, Stremmel W and Eisenbach C: Entecavir as treatment for reactivation of hepatitis B in immunosuppressed patients. World J Gastroenterol 16: 5447-5451, 2010.

30. Watanabe M, Shibuya A, Takada J, Tanaka Y, Okuwaki Y, Minamino T, Hidaka H, Nakazawa T and Koizumi W: Entecavir is an optional agent to prevent hepatitis B virus (HBV) reactivation: a review of 16 patients. Eur J Intern Med 21: 333-337, 2010. 